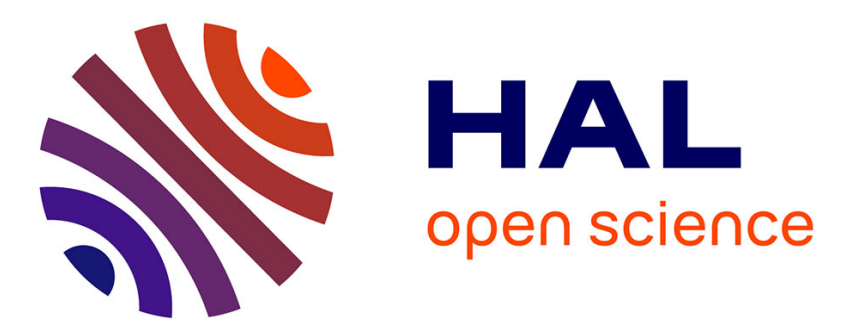

\title{
Revealing Dynamic Rotation of Single Graphene Nanoplatelets on Electrified Microinterfaces
}

Andrew Pendergast, Zejun Deng, Fouad Maroun, Christophe Renault, Jeffrey Dick

\section{- To cite this version:}

Andrew Pendergast, Zejun Deng, Fouad Maroun, Christophe Renault, Jeffrey Dick. Revealing Dynamic Rotation of Single Graphene Nanoplatelets on Electrified Microinterfaces. ACS Nano, 2021, 15 (1), pp.1250-1258. 10.1021/acsnano.0c08406 . hal-03318198

\section{HAL Id: hal-03318198 \\ https://hal.science/hal-03318198}

Submitted on 23 Aug 2021

HAL is a multi-disciplinary open access archive for the deposit and dissemination of scientific research documents, whether they are published or not. The documents may come from teaching and research institutions in France or abroad, or from public or private research centers.
L'archive ouverte pluridisciplinaire HAL, est destinée au dépôt et à la diffusion de documents scientifiques de niveau recherche, publiés ou non, émanant des établissements d'enseignement et de recherche français ou étrangers, des laboratoires publics ou privés. 


\section{Revealing Dynamic Rotation of Single Graphene Nanoplatelets on Electrified Microinterfaces}

Andrew D. Pendergast ${ }^{1}$, Zejun Deng ${ }^{1}$, Fouad Maroun $^{1}$, Christophe Renault $^{2 *}$, and Jeffrey E. Dick $^{1,3 *}$

${ }^{1}$ Department of Chemistry, The University of North Carolina at Chapel Hill, Chapel Hill, NC 27599

${ }^{2}$ Physique de la Matière Condensée, CNRS, Ecole Polytechnique, 91128 Palaiseau, France ${ }^{3}$ Lineberger Comprehensive Cancer Center, The University of North Carolina at Chapel Hill, Chapel Hill, NC 27599

*To whom correspondence should be addressed: jedick@email.unc.edu, christophe.renault@polytechnique.edu

TOC Figure
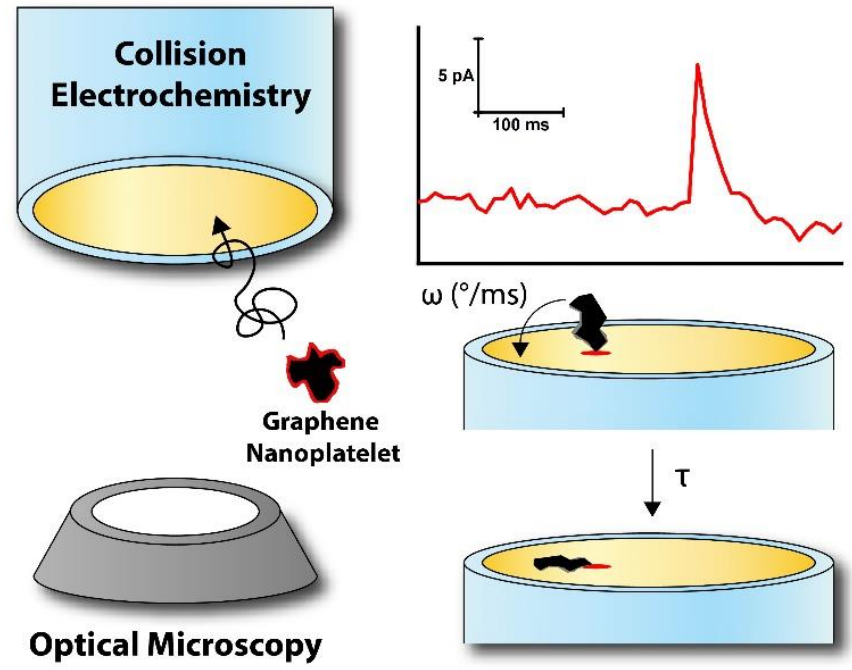


\begin{abstract}
Nanoparticles interact with a variety of interfaces, from cell walls for medicinal applications to conductive interfaces for energy storage and conversion applications. Unfortunately, quantifying dynamic changes of nanoparticles near interfaces is difficult. While optical techniques exist to study nanoparticle dynamics, motions smaller than the diffraction limit cannot be quantified. Single entity electrochemistry has high sensitivity, but the technique suffers from ambiguity in the entity's size, morphology, and collision location. Here, we combine optical microscopy, single entity electrochemistry, and numerical simulations to elucidate the dynamic motion of graphene nanoplatelets at a gold ultramicroelectrode (radius $\sim 5 \mu \mathrm{m}$ ). The approach of conductive graphene nanoplatelets, suspended in $10 \mu \mathrm{M} \mathrm{NaOH}$, to an ultramicroelectrode surface was tracked optically during the continuous oxidation of ferrocenemethanol. Optical microscopy confirmed the nanoplatelet size, morphology, and collision location on the ultramicroelectrode. Nanoplatelets collided on the ultramicroelectrode at an angle, $\theta$, enhancing the electroactive area, resulting in a sharp increase in current. After the collision, the nanoplatelets reoriented to lay flat on the electrode surface, which manifested as a return to the base-line current in the amperometric $i$-t response. Here, we report the coupled optoelectrochemical observation of single graphene nanoplatelet collision events under amperometric conditions. Through correlated Multiphysics simulations, the dynamics of these collision events and subsequent reorientation processes can be extracted to reveal angular velocity on the order of 0.5 to $2 \% \mathrm{~ms}$. This simulation driven approach can be extended to a wide range of asymmetric single entities including nanorods, planar nanoflakes, and nanoparticle clusters for robust and unambiguous determination of collision dynamics on electrified interfaces one entity at a time.
\end{abstract}

Keywords: Single entity, collision electrochemistry, graphene nanoplatelet, correlated measurement 


\section{Introduction}

Nanoparticles often reveal desirable physicochemical properties compared to their bulk material counterparts including enhanced reactivity, stability, and surfacearea-to-volume ratios for applications ranging from biomolecule sensing to fuel cell electrocatalysis. ${ }^{1-4}$ For practical applications, these nanomaterials must be supported on a scaffolding material, such as inert graphitic carbon or biomolecule assemblies, to amplify the activity of a single nanoparticle for detection or generation..$^{5-7}$ Nanoparticles and other single entities adsorbed on interfaces are not always static. ${ }^{8}$ For example, enzymatic fuel cells have been shown to demonstrate orientation-specific performance characteristics, ${ }^{9}$ necessitating techniques to control and monitor surface morphology at the single entity level. Recently, extracellular vesicles have been shown as promising avenues for targeted biomolecule delivery in biological systems, ${ }^{10}$,

11 motivating studies of single vesicle dynamics during exocytosis processes. ${ }^{12,13}$ For complex populations of nanomaterials, the ability to understand and control interfacial geometry offers the exciting prospect of tunable performance based on orientation. Microscopic techniques offer a powerful method to probe structural and chemical information for biomolecules, nanoparticles, and living cells with excellent spatial resolution, even revealing fundamental dynamic behavior of fluorescent molecules. ${ }^{14,}{ }^{15}$ By coupling the high temporal resolution of electrochemical measurements and the excellent spatial resolution of microscopy, correlated measurements can be obtained to provide direct insight on nanointerfaces in real-time, opening avenues to control the chemical properties and performance of nanoparticles with unprecedented precision.
The measurement of single entities by electrochemical collision techniques has emerged as a powerful analytical method to directly probe heterogeneities within complex nano-object populations. ${ }^{16,} 17$ These techniques are very sensitive, as groups have reported the collision of single enzyme molecules and atoms on ultramicro- and nanoelectrodes, ${ }^{18-21}$ and groups have studied the bouncing of single nanoparticles on ultramicroelectrode surfaces. ${ }^{8}$ Amperometric and voltammetric collision techniques have been applied to a range of single entity populations, revealing variations in physicochemical properties as a function of entity size, morphology, and location on an underlying electrode. ${ }^{22-25}$ In single entity electrochemistry experiments, entities diffuse or migrate through solution and collide with an ultramicroelectrode, generating a change in current or potential depending on the technique being used to observe the collision..$^{26-28}$ Collision electrochemistry has been previously reported to study a range of nano-entities including single metallic nanoparticles, emulsion droplets, biological vesicles, and single cells. ${ }^{12,}{ }^{20,29-31}$ While these studies can illustrate the inherent polydispersity in physicochemical properties for heterogeneous systems, it is often difficult to decouple the chemical properties of the colliding entity and the physical properties of the system (i.e., entity size, entity morphology, and where on the ultramicroelectrode the entity collides). ${ }^{32}$ Importantly, the enhanced mass transport at the edges of an ultramicroelectrode due to radial diffusion can result in a range of collision transient magnitudes, even for a homogeneous population. In most of these experiments, the colliding species has been spherical or assumed spherical such that entity orientation can be ignored in the analysis. ${ }^{21,} 33$ However, not all nanoparticles of interest are perfectly symmetric, and these types of species present interesting challenges in single entity electrochemistry.

Recently, graphene nanoplatelets have emerged as a robust scaffolding material for 
applications in nanoparticle and nanowire catalysis due to the high surface-area-to-volume ratio, desirable $s p^{2}$ hybridized carbon sites, and excellent stability. ${ }^{34-37}$ As a two-dimensional material with two spatial dimensions in the micrometer range and one spatial dimension in the nanometer range, these platelets demonstrate significant polydispersity in size and morphology in addition to rotational asymmetry. ${ }^{38}$ Therefore, the collision response for a single colliding graphene nanoplatelet must be described by a combination of the size and morphology of the colliding entity, the location of the collision on the ultramicroelectrode, and the orientation of the platelet during collision. Decoupling the effects of collision dynamics and entity geometry is difficult to achieve solely through electrochemistry. Numerical models can be simulated to decouple the edge effect and amperometric responses of colliding entities; however, heterogeneous populations pose a significant challenge due to convolution of the edge effect and polydisperse colliding entities. ${ }^{39}$ Work by Renault and coworkers has provided a method to overcome the edge effect by developing hemispherical ultramicroelectrodes with constant mass transport across the entire electrode surface. ${ }^{40}$ However, the use of a hemispherical electrode does not yield size and morphology information about the colliding, asymmetric entity. Previously, Fosdick and coworkers directly characterized the edge effect at an ultramicroelectrode by correlating single entity blocking experiments with fluorescent microscopy with fluorescently labelled polystyrene beads. ${ }^{41}$ Recently, Thorgaard and coworkers employed correlated optoelectrochemical measurements to probe the effects of electroosmotic flow in stochastic collisions of single bacterium, demonstrating a theory driven approach to deconvoluting complex current-transient events at ultramicroelectrode surface. ${ }^{42}$ By combining the excellent temporal resolution of electrochemistry with the spatial resolution of optical microscopy, extensive characterization of single colliding entities can be achieved to begin understanding the collision dynamics for non-spherical systems.

In this article, we couple correlated single entity electrochemistry and optical microscopy measurements with finite element modelling simulations to elucidate dynamic motions of graphene nanoplatelets on ultramicroelectrodes and extrapolate a single nanoplatelet's rotational velocity after colliding with the ultramicroelectrode surface. When ferrocenemethanol oxidation is driven at the ultramicroelectrode surface and a conductive graphene nanoplatelet collides, the effective electroactive surface increases, causing a rapid increase in current. From optical microscopic measurements, these graphene nanoplatelets collides at an angle. After colliding at an angle, the graphene nanoplatelet rotates towards a parallel orientation on the ultramicroelectrode surface, and the current rapidly returns to baseline. The temporal response between the initial collision and the decrease in current attributed to platelet reorientation to lay flat on the electrode surface allows access to the angular velocity of a single graphene nanoplatelet. In this system, the graphene nanoplatelet functions as a nanoscale probe to characterize electrical and chemical forces at the ultramicroelectrode surface through correlated observation and numerical simulation. With the coupling of optical and electrochemical methods, the physical and chemical environments of these single entity 'tracers' can be known with relatively high precision, allowing extensive modelling of nanoscale reorientation events at the single entity level. Further, through the strengths of numerical simulation methods, this technique offers a platform to probe fundamental dynamic processes at a wide range of nanoscale entities including single nanoparticles, cells, and enzymes to directly relate structure/orientation/function with coupled optoelectrochemical experiments and numerical simulations. 
Opto-electrochemical monitoring of graphene nanoplatelet adsorption.

Single graphene nanoplatelet irreversible adsorption events at electrified gold ultramicroelectrodes were monitored using a home-built opto-electrochemical setup to simultaneously record optical microscopy and electrochemistry at the electrode surface, as represented schematically in Figure 1A. Optical microscopy videos were captured under bright-field illumination with an inverted microscope equipped with a $40 \mathrm{X}$ objective at a frame rate of 100 fps. The microscope was focused on the surface of a 5 $\mu \mathrm{m}$ radius gold ultramicroelectrode located approximately $1 \mathrm{~mm}$ above a glass coverslip (150 $\mu \mathrm{m}$ thick) suspended in a solution of 1 $\mathrm{mM}$ ferrocenemethanol and $100 \mu \mathrm{g} / \mathrm{mL}$ of graphene nanoplatelets with $10 \mu \mathrm{M} \mathrm{KOH}$. The average diameter of these suspended graphene nanoplatelets has been previously reported as $4.2 \pm 2.5 \mu \mathrm{m}$ characterized through SEM. ${ }^{43}$ Electrochemical currents passing through the gold surface and any adsorbed graphene nanoplatelets were recorded at $100 \mathrm{~Hz}$ and synchronized with optical microscopy video capture. A schematic representation of a single nanoplatelet collision event with simultaneous optical and electrochemical measurement is presented in Figure 1B. In brief, the Au ultramicroelectrode was biased at a potential sufficiently positive (ie. +400 $\mathrm{mV}$ vs. $\mathrm{Ag} / \mathrm{AgCl}$ ) to oxidize ferrocenemethanol at the steady-state mass transport limit and the electrode surface was simultaneously illuminated with incident light $(\lambda=425 \mathrm{~nm})$ represented by the blue arrows in Figure 1B. Upon collision, the nanoplatelet adsorbs at the electrode surface and becomes polarized to the applied bias, after which ferrocenemethanol oxidation can occur on both the electrode surface and the graphene
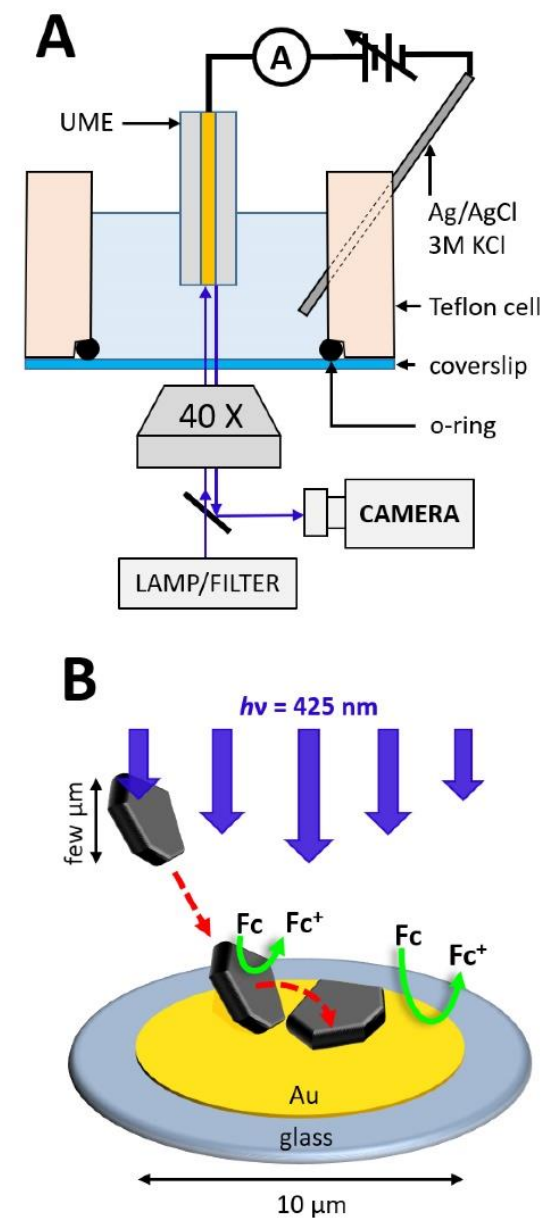

Figure 1 | Experimental Scheme. (A) Instrumental setup of home-built correlated optoelectrochemical cell for simultaneous video microscopy and amperometric observation of single graphene nanoplatelet collision events at a Au ultramicroelectrode surface. (B) Schematic representation of a graphene nanoplatelet adsorption event in the presence of ferrocenemethanol, generating a current transient during the rotational reorientation of the nanoplatelet.

surface, represented by the green arrows in Figure 1B. During this collision event and following adsorption, the combined rotational and translation movement of the nanoplatelet is recorded through optical microscopy, as represented by the red arrows in Figure 1B. 
A representative optical microscopy video corresponding to a single nanoplatelet adsorption is provided in the Supporting Information (SI V1-Figure 2 Panel A Video.mp4). Upon illumination, the gold electrode surface appears highly reflective, contrasted against the weakly reflective insulating glass sheath. Graphene nanoplatelets dispersed in solution similarly appear as high-contrast bright objects translating through the field-of-view at some distance above the ultramicroelectrode surface. During optical recording, nanoplatelets occasionally collide at the gold surface, resulting in reversible and irreversible collision events. These collisionadsorption events were observed in over 130 optical recordings with approximately $70 \%$ leading to an irreversible adsorption motif wherein the nanoplatelet remains on the electrode surface following collision. In this present work, we focus our attention on the irreversible adsorption case.

A set of several optical micrographs recorded during a collision event, presented in Figure 2A, show a typical irreversible adsorption event. During optical recording, the gold electrode surface appears as a bright circle (vide supra) and the colliding nanoplatelets appear darker as the platelets are tilted relative to the incident light, resulting in some loss of the reflected light. Panels (i), (ii), and (iii) show the gold surface before collision, at the moment of collision, and 210 ms following the initial collision when the graphene nanoplatelet has stopped moving, respectively. A 3D representation of the platelet on the electrode surface is provided for each of these optical micrographs (see SI section II). Analysis of several collision event videos leads to a few general observations. First, nanoplatelets generally arrive at the
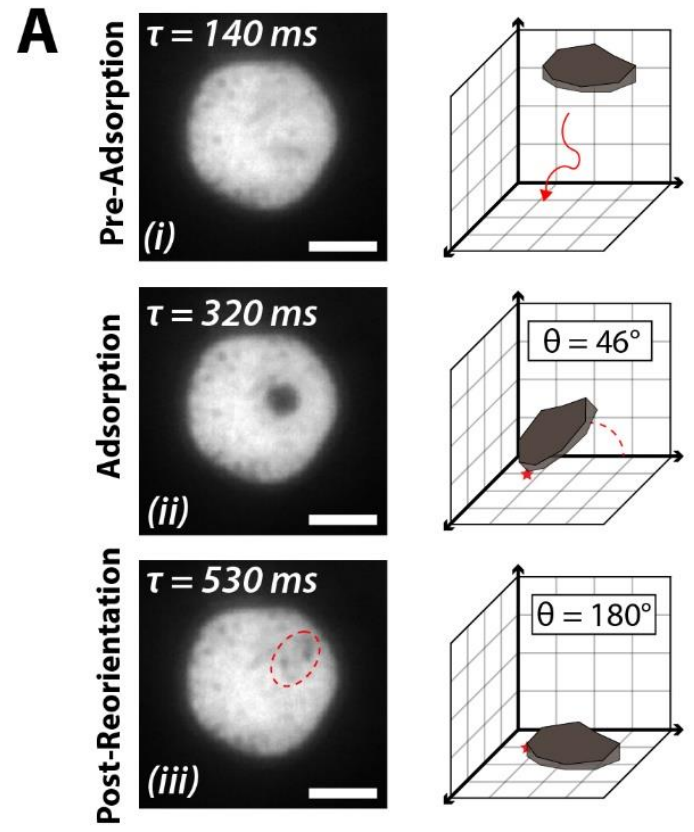

B

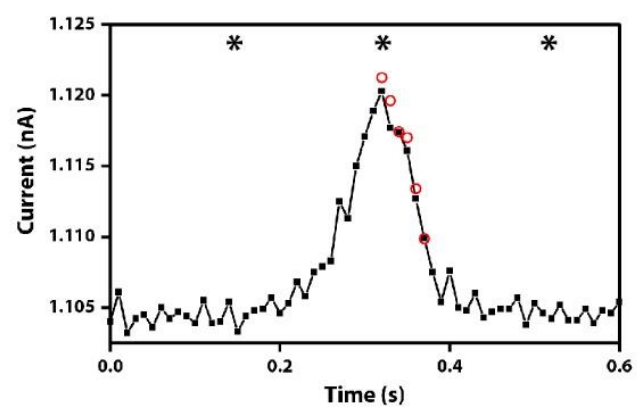

\begin{tabular}{ll|ll} 
Figure 2 & 2 & Correlated Optoelectrochemical
\end{tabular} Quantification of Angular Velocity. (A) Representative set of optical micrographs recorded during an individual collision event, with schematic representations, corresponding to (i) $180 \mathrm{~ms}$ before the collision, (ii) at the moment of collision, and (iii) $210 \mathrm{~ms}$ following collision after which the nanoplatelet lies flat on the electrode surface. (B) Complete current-time transient measured during the collision event, demonstrating a current maximum at $\mathrm{t}=320$ ms with subsequent decay to baseline current. Simulated current from optical measurements of nanoplatelet contact angle (red) show good agreement with experimental transient response. All scale bars correspond to $5 \mu \mathrm{m}$.

electrode surface with a large polar angle that is oriented with the thinnest plane-edge parallel to the electrode. It was recently shown by Thorgaard and coworkers that electroosmotic flow affect strongly the motion of micron-sized objects near an ultra- 
microelelctrode under our experimental conditions.ref In presence of this convective flow directed from the bulk toward the electrode surface, the nanoplatelets are expected to orientate their long axis in the direction of the flow (minimizing the torque) explaining the high angle of incidence observed over many collisions. Additionally, immediately following collision, the nanoplatelet begins to rotate about the plane edge in contact with the gold surface and ultimately lays down flat on the electrode surface. For the sake of simplicity, we will call this type of movement "rotation" although it should be kept in mind that the center of mass of the nanoplatelet is also translating toward the surface (i.e. movement $=$ rotation + translation). Typically, following this reorientation, the nanoplatelets remain static on the electrode surface, however, in some cases, fluctuations of the nanoplatelet position are observed in the form of "shaking" or rapid translational movements towards the edge of the electrode (see SI Movie 6). Similar "jumps" were also observed for polystyrene bead adsorbed on $\mathrm{Pt}^{41}$ and are attributed to electric potential gradients attracting anions toward the perimeter of the UME. ${ }^{32,41}$

The amperometric $i-t$ trace recorded simultaneously during the collision event shown in Figure 2A is presented in Figure 2B. In this current trace, we observe a steady state current of $\sim 1.1 \mathrm{nA}$ with a subsequent transient current spike characteristic of single entity collision measurements. Importantly, the current spike in the amperometric trace occurs simultaneously with the observed optical collision and subsequent reorientation, thereby allowing direct analytical treatment through both microscopic and electrochemical techniques. From optical microscopy, the initial adsorption contact angle can be estimated at $\sim 46^{\circ}$ with subsequent rotational motion on the order of $60 \mathrm{~ms}$. From finite element models constructed for this nanoplatelet adsorption event, the total faradaic current at the nanoplatelet/electrode surface can be simulated as a function of the polar angle between the electrode and the colliding platelet. As shown in Figure 2B, the simulated (red) and electrochemically observed (black) currents show good agreement during the rotation of a single graphene nanoplatelet following an irreversible adsorption event. We thus conclude that the decaying part of the current transient is governed by the rotation of the nanoplatelet. The current rise is under investigation.

\section{Origin of the current transient.}

The blip-type current transient observed through correlated opto-electrochemical measurements can be explained in terms of nanoplatelet rotation at the electrode surface as follows. The height of the instantaneous current spike can be related to the increased electrochemically active surface area of the nanoplatelet | electrode system following collision while the duration of the current decay can be related to the timeframe during which the nanoplatelet rotates on the electrode surface to a parallel orientation (i.e. oriented with a polar angle of $180^{\circ}$ ). Prior to a collision event, a baseline current corresponding to the transport-limited oxidation of ferrocenemethanol at the electrode surface is measured. At the moment of collision, the conductive nanoplatelet surface becomes biased at the potential of the ultramicroelectrode (graphene nanoplatelet conductivity $=2.5 \cdot 10^{6} \mathrm{~S} / \mathrm{m}$ ) such that ferrocenemethanol oxidation is observed on both the colliding nanoplatelet and the 

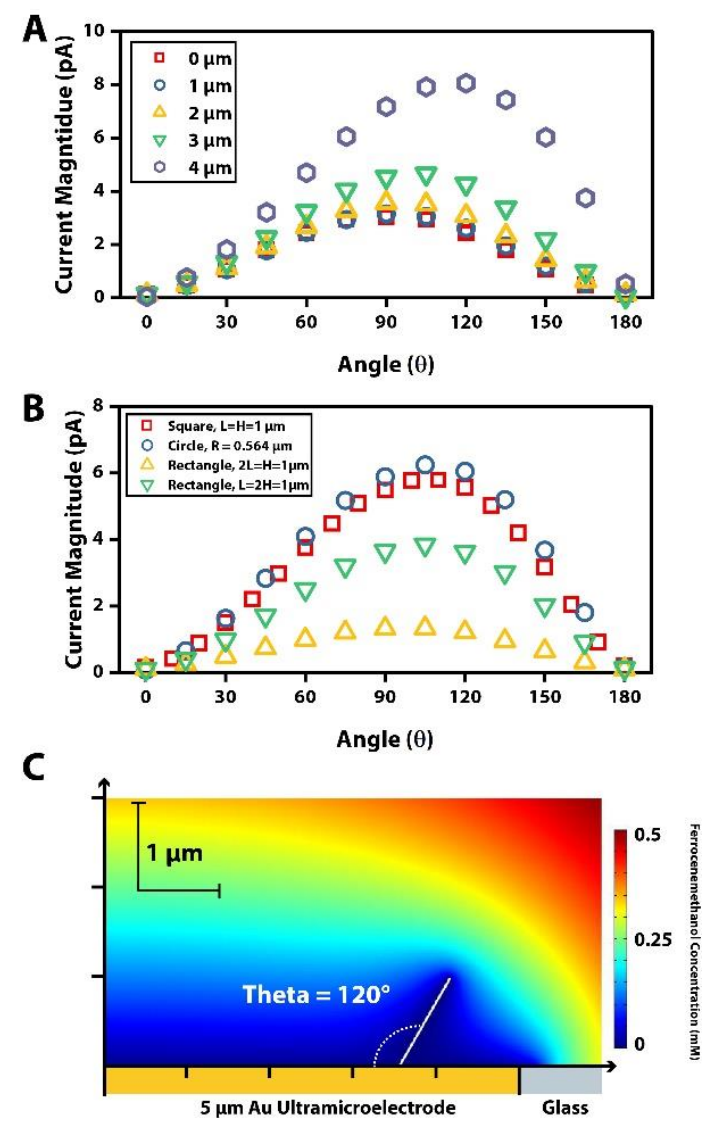

Figure 3 | Simulating Current at the NanoplateletElectrode Interface. (A) Simulated steady state currents for a $1 \mu \mathrm{m} \times 1 \mu \mathrm{m} \times 25 \mathrm{~nm}$ rectangular model graphene nanoplatelet colliding at a $5 \mu \mathrm{m}$ radius $\mathrm{Au}$ ultramicroelectrode surface at a range of collision angles and collision radial distances. Increasing collision radial distance is associated with a increase in collision angle associated with the maximum current transient magnitude. (B) Simulated steady state currents for graphene nanoplatelets of different sizes and geometries occurring at a radial distance of $r=3.5$ $\mu \mathrm{m}$. (C) Simulated concentration gradient for a $1 \mu \mathrm{m} \mathrm{x}$ $1 \mu \mathrm{m} \times 25 \mathrm{~nm}$ rectangular nanoplatelet colliding at a radial distance of $r=3.5 \mu \mathrm{m}$, demonstrating enhanced flux near the electrode edge, resulting in increased access to the bulk solution domain and thus an increased faradaic current.Tick marks on axes in panel C correspond to $1 \mu \mathrm{m}$.

underlying electrode surface. If this graphene nanoplatelet collides with a nonparallel polar angle relative to the electrode surface, the total conducting surface area (i.e. the gold electrode surface and the surface of the nanoplatelet exposed to solution) is increased, resulting in an expected increase in the total current. As the nanoplatelet progressively lays down on the surface via rotational motion, the gold surface inaccessible to the bulk solution is compensated by the conductive nanoplatelet surface. Thus, when the nanoplatelet lays flat on the electrode surface (theta $=180^{\circ}$ ), the total conducting surface area is restored to that of the pre-collision baseline, as evidenced by the current transient measurements (vide supra). In brief, we propose that the transient nature of the current transient signals is caused by the temporary increase of the electroactive surface area following the collision of a single graphene nanoplatelet on an ultramicroelectrode interface. A similar mechanism has been reported by Bard and coworkers for step-like transients of currents when electrically conducting carbon nanotubes adsorb irreversibly on a ultramicroelectrode in the presence of ferrocenemethanol. ${ }^{44}$ In their case the carbon nanotubes extend beyond the metal electrode over the glass sheath leading to a permanent increase of the area of the electrode and thus a step-current rather than a spike, termed "area amplification". We note that capacitive charging of the graphene nanoplatelet surface is expect to produce current spikes with duration on the order of hundreds of ps that are not detectable at the $100 \mathrm{~Hz}$ sampling rate used for these measurements (see SI section 8).

The magnitude and shape of collision current spikes are affected by the size and shape of the colliding graphene nanoplatelet in addition to the radial position on the ultramicroelectrode surface, the direction of reorientation, and the speed of rotation. 
Through numerical simulations, we explored the effects of size, shape, radial position on the electrode surface, and polar angle of a simulated nanoplatelet on steady-state amperometry at a nanoplateletultramicroelectrode interface. From optical measurements, nanoplatelet rotational motion is typically on the order of $\sim 0.5-2$ $\% \mathrm{~ms}$, leading to a Peclet number of approximately 0.018 (see SI section IX for further details). Thus, the diffusion of ferrocenemethanol to the nanoplateletelectrode surfaces is much faster than advection due to nanoplatelet rotation. The diffusion layer of ferrocenemethanol at the nanoplatelet-electrode surface will adapt quickly to the position and polar angle of the rotating platelet during the entirety of motion. We carried out steady-state numerical simulations at a range of polar angles to directly compute the current associated with ferrocenemethanol oxidation at the polarized nanoplatelet-electrode surface.

Figure $3 \mathrm{~A}$ shows simulated current as a function of the polar angle (theta) for a $1 \mu \mathrm{m}$ x $1 \mu \mathrm{m} \times 25 \mathrm{~nm}$ rectangular graphene nanoplatelet colliding at different radial distance. For clarity, the baseline current corresponding to the oxidation of ferrocenemethanol at a bare ultramicroelectrode has been subtracted from all simulated currents. For these simulations, a polar angle of $0^{\circ}$ and $180^{\circ}$ correspond to the nanoplatelet laying down flat on the electrode surface pointed towards the electrode center or towards the electrode edge, respectively. The current variation is bell-shaped with increasing asymmetry for nanoplatelets colliding near the edge of the electrode. While a graphene nanoplatelet falling at the center of the ultramicroelectrode produces a maximum increase of current at a normal angle with respect to the surface, the same nanoplatelet falling at $4 \mu \mathrm{m}$ from the center of the electrode will produce a maximum increase of current for a polar angle of $120^{\circ}$. The magnitude of these current transients also increases as the collision occurs further from the electrode center, shown with an increase by over a factor of two for a collision occurring $4 \mu \mathrm{m}$ from the electrode center relative to a collision occur exactly at the electrode center. Figure 3B shows the simulated steady-state current for collisions occurring at a radial distance of $4 \mu \mathrm{m}$ as a function of polar angle for nanoplatelets with different geometries. The simulated current profiles for a square and circular nanoplatelet with the same surface area are extremely close (red and blue traces). A simulated nanoplatelet collision with half the width of the original square nanoplatelet (green trace) produces a current maximum $66 \%$ that of the original nanoplatelet while a nanoplatelet with half the height of the original produces a current maximum of $23 \%$ that of the original. Importantly, the position of the current maxima and the shape of these current-angle profiles are conserved between these different geometries (see SI Figure S10). In summary, bell-shaped currenttransients are expected from collision of a graphene nanoplatelet and subsequent rotational reorientation. The magnitude of these current transients is sensitive to both the radial position of the collision on the ultramicroelectrode surface and the size of the graphene nanoplatelet. However, the shape of the current-angle profile is governed primarily by the radial position of the collision and not the shape or size of the colliding graphene nanoplatelet.

The dependence of the magnitude and shape of these current-angle profiles can be 
explained based on the overlap of diffusion layers emanating from the ultramicroelectrode and the nanoplatelet during the collision-reorientation event. A simulated concentration profile is presented in Figure 3C corresponding to the localized ferrocenemethanol

concentration profile around a $1 \mu \mathrm{m}$ square nanoplatelet colliding on the ultramicroelectrode at a radial distance of $3.5 \mu \mathrm{m}$ with a polar angle $120^{\circ}$. Near the perimeter of the ultramicroelectrode and at the edge of the graphene nanoplatelet, the concentration gradients are fairly large. can be clearly seen that the further the nanoplatelet extends in solution and the more it is able to access areas with large concentrations of ferrocenemethanol. When the nanoplatelet rotates toward the edge of the ultramicroelectrode where the gradient of ferrocenemethanol is the highest, the nanoplatelet is again able to access larger concentrations of ferrocenemethanol than it would toward the center of the ultramicroelectrode, resulting in an increased current-transient magnitude. Additional simulation details are provided in the Supporting Information section XIV, COMSOL Model Report, for a representative simulated nanoplatelet.

\section{Variability of the current transient.}
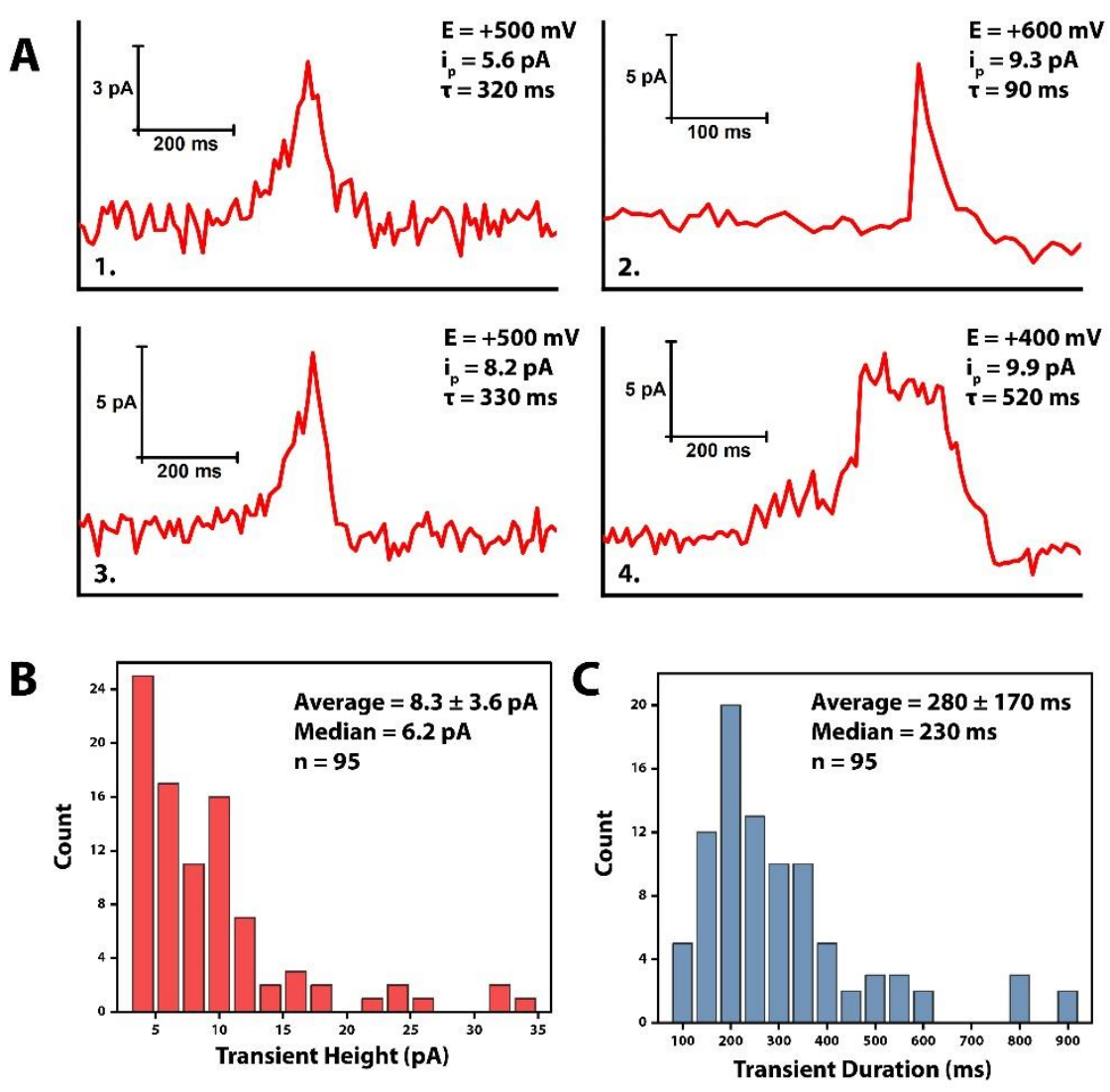

Figure 4 | Current Transients and Descriptive Statistics of Collision Events (A) Representative set of current transients associated with correlated optoelectrochemical observation of single graphene nanoplatelet collision events. Current-time traces demonstrate a wide range of magnitudes, durations, and transient shapes that can be decoupled through simultaneous measurement of nanoplatelet size, collision location, and reorientation processes at the electrode surface. (B) Histogram of current transient magnitudes for single graphene nanoplatelet collision events at potentials sufficient to oxidize ferrocenemethanol, producing an average of $8.3 \pm 3.6 \mathrm{pA}$ over 95 collision events. (C) Histogram of current transient durations for single graphene nanoplatelet collision events, producing an average of $280 \pm 170 \mathrm{~ms}$ full width at peak base over 95 collision events. Additional statistics are presented in the supporting information.

\section{Correlated opto-electrochemical}

measurements were collected for over 95 individual collision events demonstrating a large variety of current-transient characteristics that can be explained in terms of the previously described parameters. Figure 4A shows a set of representative current-time traces for individual graphene nanoplatelet adsorption events, demonstrating a range of transient 
amplitudes, durations, and shapes. For example, transient in Panel 1 is symmetrical about the current maximum while transients in Panels 2 and 3 demonstrate asymmetry about the current maximum. Further, some step-like transients were observed, as presented in Panel 4. Histograms of the duration and intensity of the transients are shown in Figure $4 \mathrm{~B}$ and $4 \mathrm{C}$, respectively. Observed magnitudes varied between 2.4 and $33.8 \mathrm{pA}$ with an average magnitude of $8.3 \pm$ $3.6 \mathrm{pA}$ while the transient-durations varied between 60 and $890 \mathrm{~ms}$ with an average duration of $280 \pm 170 \mathrm{~ms}$. These large dispersions in transient shape, duration, and amplitude can be easily understood in terms of the large amount of parameters that affect the current during these complex collision events; namely the position of the collision event on the ultramicroelectrode, the angular velocity of the falling nanoplatelet, the direction of motion, and the size and shape of the colliding nanoplatelet. Optical micrographs for these graphene nanoplatelet populations demonstrate significant heterogeneity in size and shape, as shown in SI Figure S3. Optical characterization of individual collision events further reveals a large dispersion in the radial position of collision and the size of the colliding graphene nanoplatelets, as shown in SI Figure S6 and S8. The large dispersion over multiple parameters prevents a simple correlation between the magnitude or duration of the current transients with the size or position of the graphene nanoplatelet. This complexity can be overcome in part by coupling optical and electrochemical 
measurements to characterize as much parameters as possible for each individual nanoplatelet adsorption event.

\section{In-situ measurement of the angular velocity.}

The angular velocity, or the rotation along the polar angle relative to the electrode surface, can be estimated for individual colliding graphene nanoplatelets by coupling the electrochemical transient current decay, optical characterization of the collision location, and insight from numerical simulations. As previously mentioned, numerical simulations of the steady state flux to the nanoplatelet/electrode surface following collision reveal current amplitude sensitivity to the size of the colliding graphene nanoplatelet and the location of the collision. However, by normalizing these steady-state currents to the maximum steadystate current at a given collision location, the resultant normalized current-collision angle plot is apathetic to the size or geometry of the colliding graphene nanoplatelet (see SI Section X).

For nanoplatelet collisions occurring at more than a micrometer away from the perimeter of the electrode, the maximum of the current transient is expected to occur for an angle of incidence of about $90^{\circ}$ (see Figure 3A).

From optical microscopy, the radial position of the nanoplatelet, the direction of
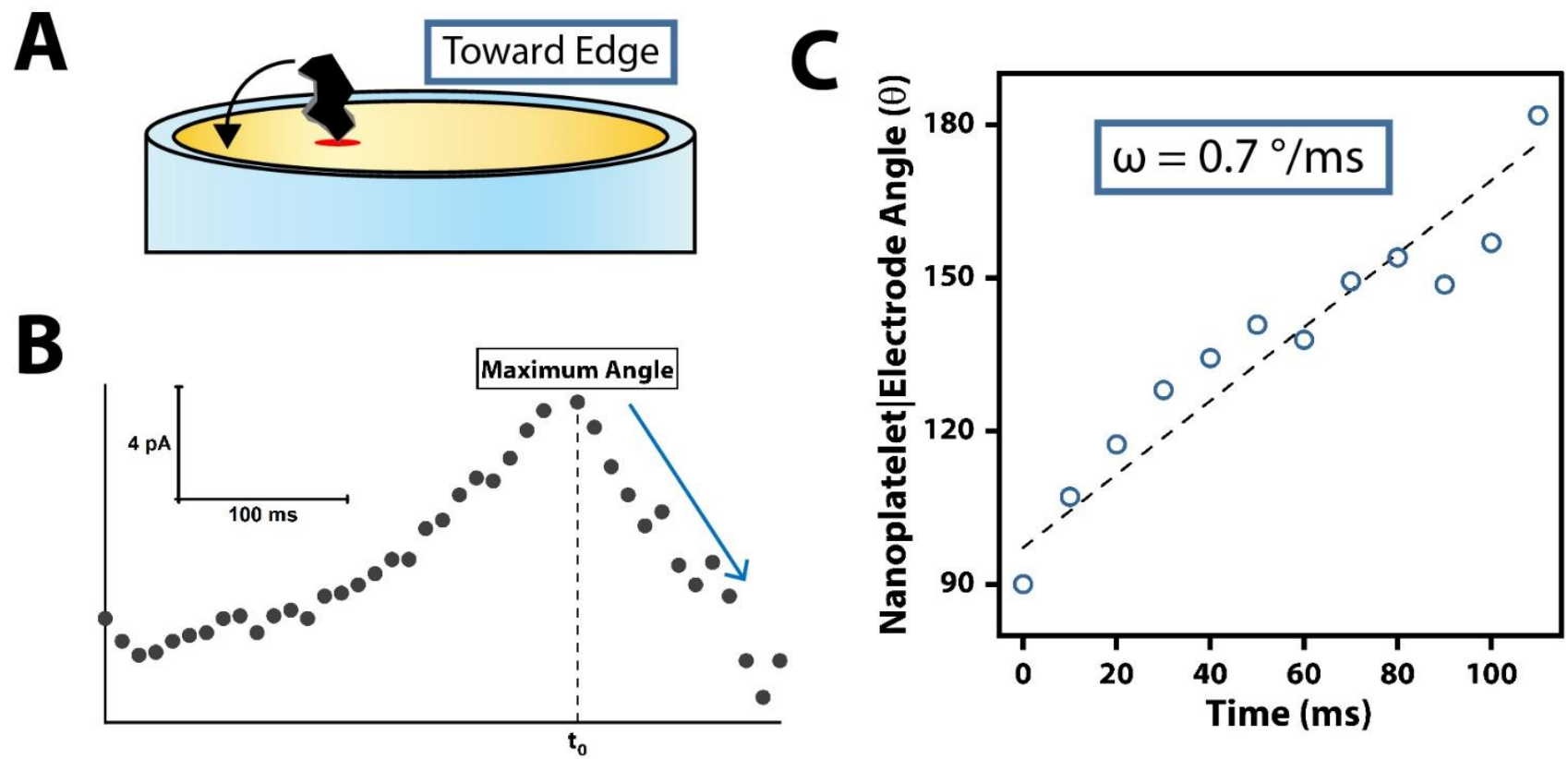

Figure 5 | Extracting Angular Velocity from Optoelectrochemical Measurements (A) Schematic representation of reorientation process on the electrode surface. From correlated optoelectrochemical measurements, the position, size, and reorientation direction of the colliding nanoplatelet can be directly observed, eliminating ambiguity associated with solely electrochemical measurement. (B) Current-time transient associated with the collision of a $0.742 \mu \mathrm{m}^{2}$ nanoplatelet at a radial distance of $1.98 \mu \mathrm{m}$ with a current peak height of $10.6 \mathrm{pA}$. By normalizing the current transient to this current maximum, the angular velocity can be extracted independent of nanoplatelet geometry. (C) From the simulated steadystate current-angle curves extracted at a collision radial distance of $2 \mu \mathrm{m}$, the normalized current transient can be converted to a collision angle transient and the average angular velocity calculated from a linear-least squares regression, providing a value of $\omega=0.7 \% \mathrm{~ms}$. 
reorientation can be directly observed, represented schematically in Figure 5A for reorientation towards the electrode edge. By fitting the normalized-current transient for a collision occurring at a known radial distance on the electrode surface to the simulated normalized-current contact angle plot at that same radial distance, the angular velocity can be estimated directly from correlated optoelectrochemical measurements and numerical simulations. As shown in Figure $5 \mathrm{~B}$ for a collision event occurring near the electrode center at a radial position $r=1.98$ $\mu \mathrm{m}$, the transient shows an increase to a maximum contact angle followed by a rapid rotation to lay flat on the electrode surface. By fitting this normalized transient decay (blue arrow in Figure 5B) to numerical simulations for nanoplatelet/electrode angles at a collision distance $r=2 \mu \mathrm{m}$ (blue points), the average angular velocity for this collision event can be extracted (black dashed line) and calculated as $0.7 \% \mathrm{~ms}$, as shown in Figure 5C. Interestingly, for collisions occurring closer to the electrode edge, at a radial position $r=3.5$ $\mu \mathrm{m}$, the average angular velocity was calculated as $1.4 \pm 0.2 \%$ ms (see SI Section XI), suggesting that nanoplatelets approaching closer from the edge of the electrode rotate faster than nanoplatelets landing near the center of the same electrode.. Recently, work by Thorgaard has demonstrated the effects of electroosmotic flow and electric fields during stochastic collision processes,${ }^{42}$ potentially providing insight for the observations of this work relating angular velocity and collision location via the presence of electroosmotic forces that are not presently considered in the numerical simulations. Future work will explore the role of electroosmotic flow in graphene nanoplatelet reorientation processes at ultramicroelectrode surfaces and the role of bipolar redox cycling at the nanoplatelet/electrode interface on the rise-times associated with these irreversible adsorption events.

\section{Conclusions}

Here, we demonstrate that coupling single entity electrochemistry and optical microscopy with Multiphysics simulations allows insight into dynamics of nanoplatelets at an electrified ultramicroelectrode surface. During the collision event, the current transient response can be described by the instantaneous increase in electrochemically active surface area following the formation of electrical contact between the electrode and the conductive colliding platelet. In the case where the platelet does not visibly migrate away from the electrode surface, we attribute the subsequent transient baseline decay to the rotation of the colliding graphene nanoplatelet to ultimately lay flat on the electrode surface. From steady-state simulations for the flux of ferrocenemethanol to the electrode|nanoplatelet interface, the observed current transients can be explained in terms of the size of the colliding platelet, the location of the collision, and the transient angle during reorientation processes. We estimate the angular velocity of micron-sized graphene nanoplatelets colliding an electrified interface to be relatively independent of the potential and on the order of few $\% / \mathrm{ms}$. The sensitivity and time resolution of optoelectrochemistry coupled with numerical simulation methods should enable the observation of sub-micron sized entities at electrode surfaces for fundamental studies at a range of entity geometries. From this work, the dynamics of single entity adsorption events can be probed through correlated optoelectrochemical and simulation methods for a wide range of single entities. This type of study would allow insight in the effects of size, orientation, and local physicochemical environment on single entity activity for systems where a single entity adsorption event offers a coupled optoelectrochemical signal, providing novel characterization methods of single entity populations. Our results demonstrate the sensitive power of electrochemistry coupled to optics and 
finite element simulations to elucidate dynamic motions at electrified micro interfaces.

\section{Materials and Methods}

Electrode Fabrication: Inlaid disk gold ultramicroelectrodes were fabricated by heatsealing $5 \mu \mathrm{m}$ radius $\mathrm{Au}$ wire (hard tempered, Goodfellow) inside a borosilicated glass capillary ( $2 \mathrm{~mm}$ outer diameter, $1.16 \mathrm{~mm}$ inner diameter, Sutter Instrument, Novato U.S.A) using an inhouse induction heating coil. The sealed Au disk electrode was subsequently polished with abrasive polishing pads $(600,800,1200$ grit) and alumina slurry $(1,0.3$, and $0.05 \mu \mathrm{m}$, Buehler, Lake Bluff, U.S.A.) until a mirror-like surface was observed under optical microscopy. Connection was made to the $\mathrm{Au}$ wire via a tungsten wire $(0.25 \mathrm{~mm}$ diameter ChemPure, Karlsruhe, Germany) and conductive silver paste (RS Components, Northants, U.K.).

Chemicals: Graphene oxide in water suspension (4 mg/mL) was purchased from Graphenea Inc. (Cambridge, USA). Ferrocene methanol, sodium hydroxide, $96 \%$ ethanol, $98 \%$ sulfuric acid and $30 \%$ hydrogen peroxide were purchased from Sigma-Aldrich (Saint Louis, USA). All chemicals were used without further purification. DI-water (18.2 M $2 . \mathrm{cm}, 2-4 \mathrm{ppb}$ total organic content) was produced with a Milli-Q Advantage A10 (Millipore) purification system. PTFE Syringe filters with a pore size $0.1 \mu \mathrm{m}$ were purchased from Merck Millipore.

Electrochemical Experimental Setup: All electrochemical measurements were carried out with a two-electrode setup represented with a potential waveform is applied between the two electrodes with the analogue potential output of a National Instrument USB 6212 acquisition card. The current is measured using a DDPCA-300 (Femto, Germany) variable gain trans-impedance amplifier. The gain of the amplifier and the output voltage are set/read with the National Instrument card. A relay placed between the reference electrode and the National Instrument card is controlled by the card and can be used to open the circuit after an experiment. A home-made
Labview code (Labview 2013 Pack 1, National Instruments) installed on a PC is used to control the National Instrument card, record the data and plot them. Typical experiments involve a current in the nA range and thus a gain of $10^{-9} \mathrm{~V} / \mathrm{A}$ was set. The data are acquired at $150 \mathrm{~Hz}$ (the bandwidth of the amplifier) and averaged in order to plot a point every $10 \mathrm{~ms}$.

Graphene Nanoplatelet Stock Preparation: A $100 \mu \mathrm{g} / \mathrm{mL}$ stock solution of graphene nanoplatelets in $10 \mu \mathrm{M} \mathrm{NaOH}$ was prepared and the stock solution was sonicated for 10 minutes prior to collision measurements. Following injection into the electrochemical cell, the solution was manually triturated to ensure homogenization.

Correlated Optical Microscopy: Correlated optical microscopy measurements were collected in reflectance with an inverted microscope (IX 72, Olympus) equipped with $40 \mathrm{X}$ objective (XXX). The epi-illuminationwas done with a Halogen light source (XXX) with a blue optical filter (D425/60X Notch filter, Chroma) to facilitate the differentiation between the single graphene nanoplatelets and the $\mathrm{Au}$ substrate background(less reflective in the blue). Correlated microscopy videos were collected at 20-40 Hz within HCImage using an external trigger controlled within Labview and image stacks were subsequently exported for analysis using ImageJ. Micrographs of single graphene nanoplatelets at the electrode interface were background subtracted relative to the electrode prior to entity collision through an ImageJ macro to emphasize surface morphology changes during the collision process.

COMSOL Multiphysics Simulation Details: Steady-state amperometry for ferrocenemethanol oxidation at the electrode | solution | nanoplatelet domain was simulated at a range of nanoplatelet geometries using COMSOL Multiphysics 5.5. For the oxidation of ferrocenemethanol $(\mathrm{FcMeOH})$, an uncharged outer sphere redox molecule, to ferroceneiummethanol ( $\mathrm{FcMeOH}+)$, 
the complete electrochemical reaction can be written as:

$$
\begin{aligned}
\text { FcMeOH }-e^{-} & \rightleftarrows F C M e O H^{+} \mid E^{0} \\
& =0.22 \mathrm{~V} \text { vs. Ag/AgCl}
\end{aligned}
$$

Under diffusion control, the flux of $\mathrm{FcMeOH}$, $J_{\mathrm{FCMeOH}}$, can be calculated from Fick's first law as:

$$
J_{F C M e O H}(x, t)=-D_{F C M e O H} \frac{\partial C_{F C M e O H}(x, t)}{\partial x}
$$

Where $D_{\mathrm{FCM} \text { OOH }}$ is the diffusion coefficient of $\mathrm{FcMeOH}$ in water, and $C_{\mathrm{FCMeOH}}(x, t)$ is the concentration of $\mathrm{FcMeOH}$. Additionally, from Fick's second law, the concentration gradient with respect to time can be written as:

$$
\frac{\partial C_{\mathrm{FCMeOH}}(x, t)}{\partial t}=D_{\mathrm{FcMeOH}}\left(\frac{\partial^{2} C_{\mathrm{FCMeOH}}(x, t)}{\partial x^{2}}\right)
$$

At insulating boundaries, a zero-flux condition was imposed such that:

$$
J_{\mathrm{FCMeOH}}(x, t)=0
$$

At the nanoplatelet | Electrode surface, the total flux can be modelled by Butler-Volmer kinetics, providing flux, $J_{\mathrm{FcMeOH}}$, as:

$$
\begin{aligned}
J_{\mathrm{FCMeOH}}=-k^{0}[ & C_{\mathrm{FCMeOH}}(x, t) e^{-\alpha f \eta} \\
& \left.-C_{\mathrm{FCMeOH}+}(x, t) e^{(1-\alpha) f \eta}\right] \\
& =-J_{\mathrm{FCMeOH}+}
\end{aligned}
$$

Where $k^{0}$ is the heterogeneous rate constant, $\alpha$ is the transfer coefficient, $f$ is $\mathrm{F} / \mathrm{RT}$, and $\eta$ is the overpotential. From the flux, the current magnitude can be calculated as:

$$
|i|=F \bullet J_{F C M e O H}
$$

By integrating the total normal flux to the electrode and nanoplatelet surfaces under steadystate simulation conditions, the total current under a given set of conditions can be extracted.

The bulk solution domain was simulated using a three-dimensional geometry as a hemisphere with a radius of $750 \mu \mathrm{m}$. The electrode was modelled as an inlaid disk electrode with a radius of $5 \mu \mathrm{m}$ centered at the axis of symmetry for the solution domain. The concentration of ferrocenemethanol at the edge of the edges of the bulk solution domain was set to $1 \mathrm{mM}$ or $1 \mathrm{~mol} / \mathrm{m}^{3}$ and a zero-flux boundary condition was set on the basal plane of the solution hemisphere domain to simulate the insulating glass region of an ultramicroelectrode. At the electrode surface, a graphene nanoplatelet was modelled as a $1 \mu \mathrm{m} \times 1 \mu \mathrm{m} \times 25 \mathrm{~nm}$ box at an angle $\Theta$ relative to the electrode surface (ie. $0^{\circ}$ corresponding to a platelet laying flat and pointing towards the center of the electrode, $90^{\circ}$ corresponding to a platelet perpendicular to the electrode surface, and $180^{\circ}$ corresponding to a platelet laying flat and pointing towards the edge of the electrode). The platelet was rotated along a set pivot point at a distance away from the electrode center along the horizontal x-axis. The steady-state flux of ferrocenemethanol to the electrode surface and the graphene nanoplatelet were numerically solved at a range of incident angles and collision locations relative to the electrode surface and the resultant calculated current values were used for analysis.

All simulations were conducted using the Transport of Dilute Species Module within COMSOL 5.5. A stationary solver configuration with a parametric sweep extension was used for all simulations. Computation was completed on a supercomputing cluster on a single node using 4 physical cores on $2.50 \mathrm{GHz}$ Intel processors. All data processing was completed using a computer equipped with an Intel Core i5-8400 CPU (2.80 $\mathrm{GHz}$ ) and $8 \mathrm{~GB}$ of RAM.

\section{Conflict of Interest Statement:}

The authors declare no competiting interests.

\section{Acknowledgments}

JED gratefully acknowledges the Unviersity of North Carolina at Chapel Hill for start-up funds, which supported this work. CR acknowledges the finacial support of the CNRS, the Agence Nationale de la Recherche (ANR-17-CE09-003401, "SEE"). ZD acknowledges the China Scholarship Council (201706370055).

\section{Author Contributions}

ADP performed experiments, built finite element models, and collected/processed data. ADP, CR, 
and JED analyzed data. ADP, CR, and JED wrote the manuscript. $\mathrm{ZD}$ performed preliminary experiments under the supervision of FM and CR. CR and JED supervised all aspects of the research. All authors have given approval to the final version of this manuscript.

\section{Supporting Information}

(1) Supporting Information: I)

Ultramicroelectrode Characterization, II) Micrograph Image Processing, III) Adsorbed Graphene Nanoplatelet Micrographs, IV) Complete Current Transients, V) Transients in the Absence of Graphene Nanoplatelet, VI) Additional Collision Transients, VII) Collision Event Statistics, VIII) NonFaradaic Considerations in Collision Transients, IX) The Magnitude of Measured Angular Velocities, X) Normalized Simulated Currents at Different Geometries, XI) Quantifying Angular Velocity for Multiple Adsorption Events, XII) Representative Simulation Parameters, XIII) Supplemental Video Index, XIV) COMSOL Model Report, XV) References. 


\section{References}

1. Campbell, F. W.; Compton, R. G., The use of nanoparticles in electroanalysis: an updated review. Analytical and Bioanalytical Chemistry 2010, 396 (1), 241-259.

2. Murray, R. W., Nanoelectrochemistry: Metal Nanoparticles, Nanoelectrodes, and Nanopores. Chemical Reviews 2008, 108 (7), 2688-2720.

3. Chen, P.-C.; Liu, X.; Hedrick, J. L.; Xie, Z.; Wang, S.; Lin, Q.-Y.; Hersam, M. C.; Dravid, V. P.; Mirkin, C. A., Polyelemental nanoparticle libraries. Science 2016, 352 (6293), 1565.

4. Nam, J.-M.; Thaxton, C. S.; Mirkin, C. A., Nanoparticle-Based Bio-Bar Codes for the Ultrasensitive Detection of Proteins. Science 2003, 301 (5641), 1884.

5. Schreiber, R.; Do, J.; Roller, E.-M.; Zhang, T.; Schüller, V. J.; Nickels, P. C.; Feldmann, J.; Liedl, T., Hierarchical assembly of metal nanoparticles, quantum dots and organic dyes using DNA origami scaffolds. Nature Nanotechnology 2014, 9 (1), 74-78.

6. Zhang, N.; Zhang, Y.; Pan, X.; Fu, X.; Liu, S.; Xu, Y.-J., Assembly of CdS Nanoparticles on the Two-Dimensional Graphene Scaffold as Visible-Light-Driven Photocatalyst for Selective Organic Transformation under Ambient Conditions. The Journal of Physical Chemistry $C$ 2011, 115 (47), 23501-23511.

7. Kamat, P. V., Graphene-Based Nanoassemblies for Energy Conversion. The Journal of Physical Chemistry Letters 2011, 2 (3), 242-251.

8. Robinson, D. A.; Liu, Y.; Edwards, M. A.; Vitti, N. J.; Oja, S. M.; Zhang, B.; White, H. S., Collision Dynamics during the Electrooxidation of Individual Silver Nanoparticles. Journal of the American Chemical Society 2017, 139 (46), 16923-16931.

9. Milton, R. D.; Wang, T.; Knoche, K. L.; Minteer, S. D., Tailoring Biointerfaces for Electrocatalysis. Langmuir 2016, 32 (10), 22912301.

10. Alvarez-Erviti, L.; Seow, Y.; Yin, H.; Betts, C.; Lakhal, S.; Wood, M. J. A., Delivery of siRNA to the mouse brain by systemic injection of targeted exosomes. Nature Biotechnology 2011, 29 (4), 341-345.

11. Blanazs, A.; Armes, S. P.; Ryan, A. J., SelfAssembled Block Copolymer Aggregates: From Micelles to Vesicles and their Biological Applications. Macromolecular Rapid Communications 2009, 30 (4-5), 267-277.

12. Dunevall, J.; Fathali, H.; Najafinobar, N.; Lovric, J.; Wigström, J.; Cans, A.-S.; Ewing, A. G., Characterizing the Catecholamine Content of Single Mammalian Vesicles by CollisionAdsorption Events at an Electrode. Journal of the American Chemical Society 2015, 137 (13), 43444346.

13. Li, X.; Dunevall, J.; Ren, L.; Ewing, A. G., Mechanistic Aspects of Vesicle Opening during Analysis with Vesicle Impact Electrochemical Cytometry. Analytical Chemistry 2017, 89 (17), 9416-9423.

14. Burnette, D. T.; Sengupta, P.; Dai, Y.; Lippincott-Schwartz, J.; Kachar, B., Bleaching/blinking assisted localization microscopy for superresolution imaging using standard fluorescent molecules. Proceedings of the National Academy of Sciences 2011, 108 (52), 21081.

15. Schermelleh, L.; Ferrand, A.; Huser, T.; Eggeling, C.; Sauer, M.; Biehlmaier, O.; Drummen, G. P. C., Super-resolution microscopy demystified. Nature Cell Biology 2019, 21 (1), 7284.

16. Baker, L. A., Perspective and Prospectus on Single-Entity Electrochemistry. Journal of the American Chemical Society 2018, 140 (46), 15549-15559.

17. Goines, S.; Dick, J., ReviewElectrochemistry's Potential to Reach the Ultimate Sensitivity in Measurement Science. Journal of The Electrochemical Society 2020, 167, 037505.

18. Dick, J. E.; Bard, A. J., Toward the Digital Electrochemical Recognition of Cobalt, Iridium, Nickel, and Iron Ion Collisions by Catalytic Amplification. Journal of the American Chemical Society 2016, 138 (27), 8446-8452.

19. Dick, J. E.; Bard, A. J., Recognizing Single Collisions of PtCl6 2- at Femtomolar Concentrations on Ultramicroelectrodes by 
Nucleating Electrocatalytic Clusters. Journal of the American Chemical Society 2015, 137, 13752-13755.

20. Dick, J. E.; Hilterbrand, A. T.; Strawsine, L. M.; Upton, J. W.; Bard, A. J., Enzymatically enhanced collisions on ultramicroelectrodes for specific and rapid detection of individual viruses. Proceedings of the National Academy of Sciences of the United States of America 2016, 113 (23), 6403-6408.

21. Glasscott, M. W.; Dick, J. E., Direct Electrochemical Observation of Single Platinum Cluster Electrocatalysis on Ultramicroelectrodes. Analytical Chemistry 2018, 90 (13), 7804-7808.

22. Renault, C.; Lemay, S. G., Electrochemical Collisions of Individual Graphene Oxide Sheets: An Analytical and Fundamental Study. ChemElectroChem 2020, 7 (1), 69-73.

23. Peng, Y.-Y.; Qian, R.-C.; Hafez, M. E.; Long, Y.-T., Stochastic Collision Nanoelectrochemistry: A Review of Recent Developments. ChemElectroChem 2017, 4 (5), 977-985.

24. Kwon, S. J.; Zhou, H.; Fan, F.-R. F.; Vorobyev, V.; Zhang, B.; Bard, A. J., Stochastic electrochemistry with electrocatalytic nanoparticles at inert ultramicroelectrodestheory and experiments. Physical Chemistry Chemical Physics 2011, 13 (12), 5394-5402.

25. Percival, S. J.; Zhang, B., Fast-Scan Cyclic Voltammetry Allows Determination of ElectronTransfer Kinetic Constants in Single Nanoparticle Collision. The Journal of Physical Chemistry $C$ 2016, 120 (37), 20536-20546.

26. Cheng, W.; Compton, R. G., Electrochemical detection of nanoparticles by 'nano-impact' methods. TrAC Trends in Analytical Chemistry 2014, 58, 79-89.

27. Sokolov, S. V.; Eloul, S.; Kätelhön, E.; Batchelor-McAuley, C.; Compton, R. G., Electrode-particle impacts: a users guide. Physical Chemistry Chemical Physics 2017, 19 (1), 28-43.

28. Zhou, H.; Park, J. H.; Fan, F.-R. F.; Bard, A. J., Observation of Single Metal Nanoparticle Collisions by Open Circuit (Mixed) Potential Changes at an Ultramicroelectrode. Journal of the American Chemical Society 2012, 134 (32), 13212-13215.

29. Dick, J. E., Electrochemical detection of single cancer and healthy cell collisions on a microelectrode. Chemical Communications 2016, 52 (72), 10906-10909.

30. Stuart, E.; Zhou, Y.; Rees, N.; Compton, R., Particle-impact nanoelectrochemistry: A Fickian model for nanoparticle transport. RSC Adv. 2012, 2, 12702-12705.

31. Zhang, F.; Edwards, M. A.; Hao, R.; White, H. S.; Zhang, B., Collision and Oxidation of Silver Nanoparticles on a Gold Nanoband Electrode. The Journal of Physical Chemistry C 2017, 121 (42), 23564-23573.

32. Bonezzi, J.; Boika, A., Deciphering the Magnitude of Current Steps in Electrochemical Blocking Collision Experiments and Its Implications. Electrochimica Acta 2017, 236, 252-259.

33. Quinn, B. M.; van't Hof, P. G.; Lemay, S. G., Time-Resolved Electrochemical Detection of Discrete Adsorption Events. Journal of the American Chemical Society 2004, 126 (27), 83608361.

34. Ambrosi, A.; Chua, C. K.; Bonanni, A.; Pumera, M., Electrochemistry of Graphene and Related Materials. Chemical Reviews 2014, 114 (14), 7150-7188.

35. Georgakilas, V.; Tiwari, J. N.; Kemp, K. C.; Perman, J. A.; Bourlinos, A. B.; Kim, K. S.; Zboril, R., Noncovalent Functionalization of Graphene and Graphene Oxide for Energy Materials, Biosensing, Catalytic, and Biomedical Applications. Chemical Reviews 2016, 116 (9), 5464-5519.

36. Li, Y.; Wang, H.; Xie, L.; Liang, Y.; Hong, G.; Dai, H., MoS2 Nanoparticles Grown on Graphene: An Advanced Catalyst for the Hydrogen Evolution Reaction. Journal of the American Chemical Society 2011, 133 (19), 72967299.

37. Zhang, B.; Fan, L.; Zhong, H.; Liu, Y.; Chen, S., Graphene Nanoelectrodes: Fabrication and Size-Dependent Electrochemistry. Journal of the American Chemical Society 2013, 135 (27), 10073-10080. 
38. Poon, J.; Batchelor-McAuley, C.; Tschulik, K.; Compton, R. G., Single graphene nanoplatelets: capacitance, potential of zero charge and diffusion coefficient. Chemical Science 2015, 6 (5), 2869-2876.

39. Bard, A. J. F., Larry R. , Electrochemical Methods: Fundamentals and Applications, 2nd Edition

Wiley: 2000.

40. Deng, Z.; Elattar, R.; Maroun, F.; Renault, C., In Situ Measurement of the Size Distribution and Concentration of Insulating Particles by Electrochemical Collision on Hemispherical Ultramicroelectrodes. Analytical Chemistry 2018, 90 (21), 12923-12929.

41. Fosdick, S. E.; Anderson, M. J.; Nettleton, E. G.; Crooks, R. M., Correlated Electrochemical and Optical Tracking of Discrete Collision Events. Journal of the American Chemical Society 2013, 135 (16), 5994-5997.

42. Thorgaard, S. N.; Jenkins, S.; Tarach, A. R., Influence of Electroosmotic Flow on Stochastic Collisions at Ultramicroelectrodes. Analytical Chemistry 2020, 92 (18), 1266312669.

43. Deng, Z.; Maroun, F.; Dick, J. E.; Renault, C., Detection of individual conducting graphene nanoplatelet by electro-catalytic depression. Electrochimica Acta 2020, 355, 136805.

44. Park, J. H.; Thorgaard, S. N.; Zhang, B.; Bard, A. J., Single Particle Detection by Area Amplification: Single Wall Carbon Nanotube Attachment to a Nanoelectrode. Journal of the American Chemical Society 2013, 135 (14), 52585261. 